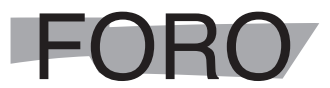

Revista de Derecho

\title{
Diversidad de familias: conformación, revolución socioeconómica y protección jurídico estatal
}

Diversity of families: conformation, socioeconomic revolution and state juridical protection

\section{José Ernesto Tapia Paredes}

Abogado, docente e investigador, Ecuador

jose.tapia@uasb.edu.ec

ORCID: 0000-0001-6466-1244

Richard Fernando Quezada Zambrano

Investigador adjunto, Ecuador

rifer10@outlook.com

ORCID: 0000-0003-2806-5589

DOI: https://doi.org/10.32719/26312484.2019.32.8

Fecha de recepción: 23 de abril de 2019

Fecha de aceptación: 4 de septiembre de 2019 


\section{RESUMEN}

El presente trabajo se centra en la diversidad de familias, que, tal como se describe, pueden iniciar tanto desde la atracción física como desde la necesidad o desde el afecto, desafiando incluso los parámetros reduccionistas y aislantes de la monogamia y el monoamor.

El contenido del artículo, sin ánimo de agotar la gran diversidad de familias que se pueden formar, presenta varias estructuras familiares a partir de una aproximación de las circunstancias de origen particular de cada una e incluye aquellas familias cuya consolidación bien podría transitar hacia elementos de amplia integración social.

Posteriormente el documento refiere varias circunstancias de represión de las que el afecto y la feminidad han sido víctimas históricas y cuyos efectos no solo se observan en el ámbito intrafamiliar sino social. Así mismo, el texto se ocupa de indicar por qué el interés conservador para mantener la estructura familiar monogámica reduccionista dominante como único modelo protegible le resulta ventajoso y conveniente al sistema de producción privado, contemplando la posibilidad de revolucionar la economía a diferentes niveles a partir de la noción de familia.

Finalmente, este trabajo realiza una rápida referencia del alcance de la obligación jurídica que el Estado ecuatoriano posee respecto a garantizar la consecución de los fines de la diversidad de familias que puedan constituirse.

PAlABRAS Clave: Diversidad de familias, monogamia, monoamor, poligamia, poliamor, pansexualidad, afectividad y feminidad.

\section{ABSTRACT}

This work focuses on the diversity of families that can start from physical attraction, or from necessity, or from affection, defying even the reductionist and isolating parameters of the monogamy and the monolove.

The content of the article without the intention of deplete the great diversity of families that can be formed, presents several family structures from an approximation of the circumstances of particular origin of each one and includes those families whose consolidation could well move towards elements of broad social integration.

Subsequently, the document refers to several circumstances of repression in which affection and femininity have been historical victims and whose effects are not only observed in the field intrafamily if not social. Likewise, the text is concerned with indicating why the conservative interest in maintaining the dominant reductionist monogamous family structure as the only protectable model it is advantageous and convenient for the private production system, contemplating the possibility of revolutionizing the economy at different levels based on the notion of family. 
Finally, this work makes a quick reference regarding the scope of the legal obligation that the Ecuadorian State has regarding guaranteeing the achievement of the purposes of the diversity of families that may be constituted.

KeYwords: Diversity of families, monogamy, monolove, polygamy, polylove, pansexuality, affectivity and femininity.

Vivimos en un mundo en el que nos escondemos para hacer el amor... pero la violencia se practica a plena luz del día.

John Lennon

\section{CONFORMACIÓN DE FAMILIAS}

$\mathrm{E}$ 1 estereotipo de la familia monogámica representado en la imagen de una pareja formada solamente por una mujer y un hombre que unen sus posesiones materiales para emprender en una vida conjunta, así como sus capacidades reproductivas para procrear hijos, ha dejado de ser la única imagen posible de la familia que debe protegerse por parte de los Estados. Este estereotipo de familia que había sido el modelo ideológico de una determinada cultura y que se impuso por mucho tiempo ante la diversidad de la naturaleza y de las expresiones humanas, presencia en la actualidad la reemergencia de otras formas de conformar familias, afectos y vínculos de cooperación.

Así, por ejemplo, entre las diferentes maneras en que las familias pueden formarse se encuentran aquellas que se generan por vínculos afectivos en que las personas eligieron libre y voluntariamente unir su vidas por los sentimientos que han envuelto sus relaciones humanas; también están aquellas en que las circunstancias de necesidad han dado origen a la agrupación de individuos, ya sea que sus miembros tengan o no vínculos sanguíneos o afectivos como punto de partida; de igual manera, se encuentran aquellas cuyo inicio ha ocurrido por el gusto y la atracción sexual que pudieron o no encontrar con el tiempo vínculos afectivos; aquellas que consideran e identifican en el hábitat o ambiente una madre, hermanos, padre, ancestros u otro miembro familiar haciendo que el concepto adquiera concepciones mucho más amplias y extensi$\operatorname{vas}^{1}$ que las del estereotipo restrictivo y dominante.

1. José Ernesto Tapia Paredes, "Perspectivas para la protección normativa de los conocimientos tradicionales frente al régimen de propiedad intelectual en el comercio internacional" (tesis de maestría, Universidad Andina Simón Bolívar, Sede Ecuador, 2014), 27. 
De entre las familias descritas en el párrafo anterior, existen numerosos casos correspondientes a aquellas que han iniciado por la atracción o gusto sexual; muchas de ellas formadas en nuestra región por adolescentes varones y mujeres ${ }^{2}$ desprovistos de educación sexual y familiar, por lo que no han podido prevenir embarazos no planificados, ${ }^{3}$ circunstancia que, sumada al factor cultural, ha conllevado a formalizar las relaciones sexuales no necesariamente afectivas en figuras matrimoniales tanto religiosas como estatales. ${ }^{4} 56$

Por el contrario, es muy diferente lo que sucede con la mayoría de familias homoparentales, muchas de las cuales también surgen inicialmente de la atracción sexual, sin embargo, estas familias realizan una elaborada planificación familiar y se aseguran de ser psicológica, emocional y económicamente aptas cuando desean tener hijos y se comprometen a una vida de cuidado, desarrollo y crianza, ya sea que adopten o procreen por mecanismos de reproducción asistida, lo cual además es consecuencia lógica del uso de estos mismos mecanismos.

En la adopción, por ejemplo, las parejas homosexuales se someten al cumplimiento de todas las pruebas y verificación de requisitos económicos, psicológicos, de entorno y demás para ser declarados idóneos; $\mathrm{y}$, en el mecanismo de reproducción asistida, la inversión de tiempo, esfuerzo, acuerdos legales e incluso dinero para procrear hace que estas familias se obliguen a planificar con mucha más rigurosidad el futuro en lugar de verse enfrentadas a un embarazo accidental o no planificado.

Respecto a las familias por necesidad, haciendo referencia a elementos más coyunturales en lugar de la necesidad gregaria de supervivencia, podemos observar una robusta presencia de circunstancias económicas y de migración que han creado el ambiente propicio para que se conformen familias por necesidad como aquellas for-

2. Organización Mundial de la Salud, El embarazo en adolescentes (Washington: OMS, 2015), 3 y 4. 〈https://www.paho.org/nic/index.php?option=com_docman\&view=download\&alias=714-boletin-informativo-embarazo-en-adolescentes\&category_slug=datos-y-estadisticas\&Itemid=235\& fbclid=IwAR1sz m6MG_nuQueYH91tfvicu6f56Hzzj8nyHB9pzQMqV9RVKU1z1R8pALg).

3. O. Cedre Blanco L. y M. E. Guerra, Factores determinantes del embarazo adolescente (Venezuela: Universidad Central de Venezuela, 2015), 116.

4. Héctor Fong, Dalila De la Cruz y Rocío López, Vidas robadas: repercusiones en la salud integral en las niñas menores de 14 años embarazadas (Guatemala: Observatorio de Salud Reproductiva, 2015), 20.

5. Fondo de las Naciones Unidas para la Infancia, Diez razones para poner fin legalmente al matrimonio infantil en República Dominicana (Santo Domingo: UNICEF, 2019), 3, 〈https://www.unicef.org/republicadominicana/Razones_ponerfin_MIUT_WEB.PDF?fbclid=IwAR3x-EACRZ015GTjbP83ovxuGHfPRGCvAxdQ_oYkUMG9wTXtLr0kAWry6A).

6. William Martínez, "Matrimonios a la fuerza", El Tiempo (Colombia), 5 de marzo de 2000, 〈https://www. eltiempo.com/archivo/documento/MAM-1304386? fbclid=IwAR0FGrFf6cJew1rpiPAomCIAdeGKSqPIe pl2W0mzH7Sdor_M_XXDLBHEcBg). 
madas por abuelos y nietos, tíos y sobrinos e incluso amigos agremiados con fines de mutua ayuda y cooperación libres de atracción sexual entre sí.

Ahora bien, con la breve referencia de la amplia variedad de familias que pueden constituirse tanto antes como después de actos de formalización como el matrimonio religioso o estatal, y observándose que dichos actos de formalización están presentes en algunos tipos de familia pero no necesariamente en todos, pasaremos a referirnos a aquellas familias iniciadas por afecto y no por atracción física, es decir, personas que van construyendo afectos y sentimientos mientras se van conociendo, reconociendo y apoyando en su desarrollo como individuos y unidad colectiva, sentimientos que con posterioridad se cristalizaran en una relación sexo-afectiva sin haber iniciado en la atracción física, sino en el paulatino incremento de sentimientos y afectos.

Para este momento ya debe haber quedado claro que el afecto que una persona puede sentir por otro ser humano puede surgir indistintamente de la genitalidad o rol de género tanto de quien ama como de quien es amado, y, por otro lado, también debe ser muy claro que no todo afecto tiene connotación o finalidad sexual.

Con lo dicho entonces resultará más sencillo comprender que existen seres humanos capaces de ver a otros seres humanos sin intencionalidad sexual e iniciar una relación de cordialidad y cooperación que durante su proceso va consolidando un vínculo que transciende hacia el afecto, el cual, en algunos casos, podrá expresarse en actividades sexuales así como otras expresiones, y, en otros casos, dicha expresión no aparecerá ni ocurrirá puesto que jamás identificará el afecto como atracción sexual, dándonos muestra de lo diverso que puede ser el ser humano al momento de iniciar o consolidar familias y relaciones interpersonales, por lo que se corrobora que ser o no heterosexual resulta indiferente para la entrega de afecto pleno y sincero.

Retornando al tema, es importante observar cómo el modelo de familia que la cultura dominante había señalado como único defendible ideologizó la sexualidad, y su aplastante imposición homogeneizadora trató de masificarse llegando a considerarse incluso como si se tratara de un modelo natural, y el único posible.

Este modelo de lo que se consideraba familia, al ser visto normativamente como oficial, conducía a rechazar y desproteger todas las demás estructuras que no encajaban en el estándar, habiendo limitado y cohibido a las personas que inician la conformación de una familia por afecto para que puedan formalizarse ante el resto de la sociedad, cosa que especialmente ocurría cuando las relaciones surgidas a partir del afecto no corresponden a afectos monógamos heterosexuales, sino a afectos poligámicos y/o homosexuales. Es decir, la naturalidad con la que surgen los afectos ha sido por siglos reprimida y llevada a las zonas de informalidad y oscuridad gracias a un prototipo rígido de familia estándar monogámico y monoamoroso. 
No obstante, una buena parte del mundo se ha resistido al modelo monogámico y considera que la familia puede también estar representada en estructuras poligámicas; así sucede en la actualidad en países como Afganistán, Argelia, Baréin, Bangladés, Benín, Birmania, Burkina Faso, Camerún, Catar, Chad, Congo, Egipto, Emiratos Árabes Unidos, Gabón, Gambia, India, Indonesia, Irak, Irán, Jordania, Kuwait, Líbano, Liberia, Libia, Malasia, Malí, Mauritania, Níger, Nigeria, Omán, Pakistán, República Centroafricana, Senegal, Sierra Leona, Somalia, Tanzania, Túnez, Togo, Uganda, Yemen, Yibuti, Zaire y Zambia, y países europeos como Reino Unido, Países Bajos, Suecia y Francia reconocen los matrimonios poligámicos en determinadas circunstancias; además de que, en casos como el de Kenia, el concepto de poligamia rebasa la figura del hombre con varias mujeres, puesto que la mujer también está facultada por la sociedad a estar en unión con más de un hombre. ${ }^{7}$

En este aspecto, la poligamia, igual que la monogamia, puede originarse con mucha diversidad. Puede surgir del afecto, de la atracción física, de la necesidad e incluso del mero compromiso entre familias para casar a uno de sus hijos o hijas.

Por ello, sin negar la presencia de las antes referidas familias cuyo punto de origen es la atracción sexual o la necesidad de cooperación, también tenemos las surgidas por la afectividad tanto monoamorosa como poliamorosa. El concepto de pansexualidad puede ayudar a clarificar el escenario.

Venido del prefijo $\pi \alpha v$ (pan) del griego antiguo que significa "todo", unido al termino "sexualidad" para hacer referencia a la orientación sexual, el término pansexual se utiliza para referirse a una persona que empatiza con todas las orientaciones sexuales, es decir, una persona capaz de sentir atracción no solo física sino afectiva por cualquier persona sin importarle su orientación sexual, el género o la genitalidad; por lo tanto, se encarna en aquellas personas que, haciendo uso de su plena madurez mental y psicoemocional, han iniciado sus relaciones principalmente desde el afecto y los sentimientos en lugar de iniciarlos desde la genitalidad o la atracción física; son por tanto individuos especialmente liberados de la programación binaria hombre-mujer como único modelo de afecto aceptable. ${ }^{8}$

Entonces, la apertura hacia la pansexualidad permite comprender que los seres humanos podemos vernos entre personas como personas que apartan el estigma de la genitalidad y el rol de género para dejar de tratar a estos aspectos como un requisito para la configuración de afectos y familias, ya que es posible reconocer liberadoramente en

7. Bruna Álvarez Mora y Silvina Monteros Obelar, Diversidad familiar: una perspectiva antropológica (Barcelona: Editorial UOC, 2019), 18.

8. Ana Elisa Sandoval, La licuefacción de la sexualidad: una aproximación a la pansexualidad en la modernidad líquida (Quito: Pontificia Universidad Católica del Ecuador, 2016), 58-9. 
los otros individuos de condiciones etarias y mentales similares, a las personalidades que generan sentimientos gratos para conformar relaciones en lugar de prestablecer exigencias físicas tanto de belleza como de genitalidad.

\section{LIBERAR LOS AFECTOS}

El comportamiento femenino ha sido durante siglos considerado como un comportamiento inferior por parte de la educación y cultura machistas; así, por ejemplo, el hombre modelo no podía mostrarse sensible ni afectivo porque podía considerársele débil. Esto ocasionó que, en la gran mayoría de relaciones familiares, el hombre en lugar de conciliar y llegar a acuerdos de convivencia armónica con los miembros de su familia imponga su voluntad y deseos por sobre los del resto de integrantes, creyéndose con autorización y derecho para ello en virtud de la misma anuencia social.

Aquel hombre que adoptaba comportamientos femeninos resultaba inadmisible para la cultura dominante; aquel hombre capaz de sensibilizarse, ${ }^{9}$ de llorar, de sentir tristeza y dolor por los problemas de otros y que se sentía impulsado a actuar no por un interés egoísta de dominar y de servirse de los demás sino de actuar por el amor y la empatía de colaborar, aquel hombre sutil y delicado que pensaba en el bienestar del resto y no en la conquista y expansión, no podía según las sociedades machistas convertirse en un jefe, un dirigente o una autoridad decisoria, ya sea que se trate del ámbito público o del entorno familiar; por ello, el hombre fue adiestrado para ser aventurero, conquistador, transgresor, impositivo, una persona capaz de tomar lo que deseara para hacer con ello su voluntad y por lo tanto capacitado para rechazar y reprimir en su interior cualquier rastro de feminidad y de afecto que pudiera debilitar su imagen pública de fortaleza y don de mando. ${ }^{10}$

Todo aspecto femenino fue minimizado en su valor social y reducido al ámbito familiar, e incluso dentro del estereotipo de familia monogámica heterosexual el valor de lo femenino fue y aún continúa siendo considerado secundario; así, las decisiones tanto de las sociedades como de las familias y hasta en los ambientes académicos y científicos debían tomarse libres de sentimientos, libres de amor, libres de compasión, libres de lo femenino.

9. Belén Pascual, Masculinidades: ¿por qué los hombres también necesitan feminismo? (Castellón: Universidad de Jaume, 2015), 43-4.

10. Victoria Gastiz, Los hombres, la igualdad y las nuevas masculinidades (Araba: Instituto Vasco de la Mujer Emakunde, 2008), 28. 
Esta ruptura del equilibrio entre lo masculino y lo femenino generó la presencia de roles rígidos que reforzaban la clasificación de los seres humanos entre los que están para mandar y los que están para obedecer o para ser sometidos, desprestigiándose a aquella persona capaz de colaborar y cooperar para el mutuo desarrollo que alentaba la redistribución tanto de responsabilidades como de beneficios debido a que dicha figura ponía y pone en cuestionamiento el estatus desigual del que se benefician los dominantes.

Los espacios políticos, económicos, profesionales, científicos, entre otros, han caído precisamente en esta ideología binaria que antepone y privilegia la masculinización de todo ser humano tanto de hombres como de mujeres y reitera el desequilibrio, relegando lo femenino a un espacio marginal y haciendo que predomine lo masculino tanto en los individuos como en la institucionalidad.

Esta construcción de la identidad masculina va mucho más allá de una simple concepción biológica, ya que está inserta en la cultura, la educación y las tradiciones que han creado una serie de roles de género que han permanecido enraizados en la sociedad durante tanto tiempo que se asumen como algo biológicamente dado. La misma sociedad es la que separa a hombres y mujeres desde que son bebés en función del sexo con el que nacen, y les crea identidades sociales, mediante educación, roles de género, actitudes y trato diferentes atribuyéndoles a los hombres cualidades, roles o responsabilidades como la autonomía, la independencia, la violencia, la agresividad, saber moverse en el entorno público y el no mostrar las emociones; mientras que a las mujeres se las estereotipa como dependencia, cuidado, tranquilidad y, en muchas ocasiones, histeria; sin embargo, todos estos atributos no son en ningún caso intrínsecos al sexo o a la genitalidad, sino que son cualidades que el patriarcado enseña a través de la educación ${ }^{11}$ y que se reproduce constantemente en las relaciones sociales.

De esta forma, la masculinización de la sociedad y de todo espacio de la vida se ha convertido en un elemento íntimamente vinculado al sistema de competencia, acaparamiento y egoísmo por los recursos, puesto que es un sistema que requiere la insensibilidad que proporciona un modelo social machista. Un sistema que necesita de profesionales con capacidad para tomar decisiones frías en las que el afecto no intervenga, con políticos agresivos que confronten a los adversarios y silencien la alternancia, científicos ávidos de dominar, controlar y utilizar al entorno para provecho del mercado, e individuos formados para apartar los sentimientos de afecto y solidaridad social de cualquier decisión política, económica, profesional y científica, así como so-

11. Pascual, Masculinidades: ¿por qué los hombres también necesitan feminismo?, 12-3. 
ciedades también formadas para aplaudir intervenciones frías consideradas científicas ajenas de la sensibilidad.

Sin embargo, si bien el modelo del macho o de lo masculino perjudica en primer lugar a las mujeres mediante diversas expresiones de discriminación, violencia y más, también son víctimas del machismo los propios hombres. Hombres por ejemplo incapaces de conectarse sensiblemente con sus hijos u otros miembros de la familia y la sociedad debido a la falta de inteligencia emocional que lleva incluso a las rupturas matrimoniales y a problemas en las relaciones interpersonales; así, también, los hombres soportan perjuicios a la salud por efecto del machismo como los indicados por la Organización Mundial de la Salud (OMS) en un informe sobre Europa, que reporta que la esperanza de vida en los hombres es inferior a la esperanza de vida en las mujeres debido, entre otros aspectos, al comportamiento considerado mayoritariamente masculino de consumir alcohol y otras sustancias, el correr más riesgos laborales, e incluso ser un $40 \%$ más propensos a muertes violentas en relación a hombres de sociedades más paritarias. ${ }^{12}$

Ahora bien, todo lo mencionado no significa que el comportamiento masculino tanto individual como social deba ser atacado al propio estilo machista de persecución, dominación e imposición, sino, por el contrario, implica más bien que la masculinidad debe reencontrarse y reequilibrarse con lo femenino dentro de un proceso de liberación y revalorización afectiva en todos los espacios de la vida, tanto del modelo de familia dominante como de otros modelos de familia alternativos y principalmente dentro de la misma sociedad en todos sus escenarios de relacionamiento.

En este proceso, las personas que han sido formadas y preparadas para reprimir su feminidad podrán reencontrarse, redescubrir y armonizarse con los afectos hacia sí mismas y los demás; es decir, un proceso en el que cada individuo se permita y se le permita ser libre para amar y expresar sentimientos tanto a nivel personal como social, rescatando por lo tanto a los afectos de la exclusión, informalidad, desvalorización y oscuridad a la que han sido relegados y que han permitido la insensibilidad humana y la satisfacción egoísta de la mera individualidad no solo en el ámbito familiar sino también colectivo, permitiéndose la humanidad desequilibrada, e ingresar a un proceso de rearmonización social.

Entonces, con personas liberadas del modelo social de interrelaciones guiadas por y hacia la conquista sexual, se posibilita la readquisición de la capacidad humana para conformar familias entre personas sin los prerrequisitos de genitalidad, sino, más

12. Organización Mundial de la Salud, The health andwell-being of men in theWHO European Region: better health througha gender approach (United Kingdom: OMS, 2018), 19-26. 
bien surgidas del afecto y mutuo consenso entre las partes con madurez mental y emocional; por lo tanto, liberar la afectividad requiere necesariamente del reequilibrio masculino y femenino de cada individuo e institución y así mismo conlleva a un sinnúmero de espacios en liberación.

\section{LA CONVENIENCIA DE UNA SOCIEDAD MONOGÁMICA EN UN SISTEMA DE PRODUCCIÓN PRIVADA}

En muchas ocasiones a lo largo de la historia humana, diferentes movimientos y culturas han practicado o se han referido a la existencia de relaciones poliamorosas. Así por ejemplo, los Mosuo de China o el propio movimiento hippie colocaron en debate la naturalidad en la conformación de familias monogámicas y poligámicas, ${ }^{13}$ remembrando las experiencias de muchas comunidades y tribus que organizan el cuidado de los hijos como una responsabilidad horizontal de tipo comunitaria y solidaria, distinguiéndolas de aquellas poligámicas verticales donde solo el hombre está autorizado para tener múltiples compañeras mientras las mujeres deben mantenerse sumisas, obedientes y dependientes de la provisión masculina.

Cuando las ideas de poligamia horizontal empezaron a reforzar al mismo tiempo la posibilidad de retomar las relaciones comunitarias y tribales posibilitadas por la existencia de familias ampliadas que redistribuyeran el trabajo y sus frutos de forma colectiva y cooperativa, rescatando el espacio de lo común mediante la ocupación tanto de lo público como de lo acumulativo, empleando además desobediencia civil y no cooperación pacífica, ${ }^{14}$ le volvió a quedar muy en claro al sistema de producción privada que la poligamia y el poliamor representaban un riesgo para el modelo de dependencia industrial.

La poligamia y el poliamor colocan en cuestionamiento la imagen de familia monogámica que hace que las personas vean a la familia como una asociación minúscula de dos individuos y sus hijos que se encuentra apartada de otras familias y que, por lo tanto, posee problemas particulares y no comunitarios que debe solucionarlos individualmente, empleándose para un tercero, ya que el espacio de cooperación colectiva es inexistente o está fuertemente reducido, restringido e incluso regulado por fuerzas externas al espacio común o comunitario.

13. Diario El Mundo recuerda que Morning Glory Zell-Ravenheart, líder neopagana del movimiento hippy californiano hacía referencia al poliamor. Sara Polo, "Poliamor", El Mundo, 12 de febrero de 2016, 〈https:// www.elmundo.es/f5/2016/02/12/56bcb4f122601dc12b8b45db.html〉.

14. Daniel García, Protesta y politica: los movimientos antiguerra en Estados Unidos (Bogotá: Universidad de los Andes, 1988), 〈file:///C:/Users/JOSE/Downloads/Dialnet-ProtestaYPolitica-2177847.pdf〉. 
Este modelo de la familia burguesa puesto en cuestionamiento surge al calor del capitalismo industrial, durante la segunda mitad del siglo XVIII y se vuelve protagonista en el siglo XIX, cuando pasa de las clases burguesas a las clases obreras, siendo naturalizado como si estuviera asentada en la propia biología humana, incluso gracias a los tratados de medicina, psicología y sociología del siglo XIX, en los que la mujer aparece biológicamente preparada para la reproducción y el hombre para la producción y provisión; así, la familia nuclear dio paso a la individualización y al rechazo del comunitarismo como si este último fuera propio de las sociedades no desarrolladas. ${ }^{15}$

La modernidad resultante de procesos como el racionalismo, la Revolución francesa, la industrialización, las nuevas tecnologías, el urbanismo, entre otras, generó cambios en la organización familiar que fueron consolidándose a lo largo de dos siglos, aproximadamente; entre dichas transformaciones destaca por ejemplo la asociación entre sexualidad y amor que se aparta del modelo de la familia campesina preindustrial que utilizaba a la elevada fecundidad como un recurso para la subsistencia, puesto que el urbanismo impone la nuclearización familiar y acentúa la distribución del trabajo fuera de casa, el fraccionamiento de la familia extensa y la disminución del número de integrantes, haciendo que la familia pierda conexión con la colectividad y dependa del salario para la subsistencia. ${ }^{16}$

En los años de 1960, la defensa del modelo de familia burgués se torna imperioso para el sistema capitalista ya que, al modelo de producción privado le resultó evidente que podía enfrentar problemas si las personas, en lugar de verse como microfamilias, empezaban a considerarse familias ampliadas capaces de formar profundos lazos no solo de agrupación por necesidad, sino lazos duraderos de afecto a partir de los cuales dejan de colaborar con una producción privada y pasan a transformar sus relaciones familiares amplias en una producción comunitaria, reduciendo su dependencia del modelo industria $1{ }^{17} \mathrm{y}$ de consumismo, ya que a la par irían incrementando sus capacidades de autosuficiencia y administración común no solo de los elementos materiales, sino también de los intangibles como el conocimiento.

Por lo tanto, los frenos no solo cultural-religiosos sino jurídicos a la poligamia debían mantenerse, y una campaña por conservar la microestructura familiar y la tradición ideológica de la monogamia requería ser impulsada con fuerza para mantener el tipo de familia que le resulta ventajoso al sistema de producción privada; de esta

15. Álvarez Mora y Monteros Obelar, Diversidad familiar: una perspectiva antropológica, 28-30.

16. Carlos Eroles, Héctor Angélico y Alberto L. Bialakowsky, Familia(s), estallido, puente y diversidad: una mirada transdisciplinaria de derechos humanos (Buenos Aires: Espacio Editorial, 2006), 37-8.

17. Claudia Mazzei, Producción y reproducción: la mujer y la división socio-sexual del trabajo (Chile: Universidad Central de Chile RUMBOS, 2013), 134. 
manera se reducían las posibilidades de un sistema de producción social en función de una familia ampliada, pero, además de los frenos jurídicos y religiosos que fortalecían la concepción de la libertad sexual no solo como un acto pecaminoso y perverso, se difundía también el mensaje de riesgo de la libertad sexual para la misma salud física impulsándose y convirtiéndola en una dramática realidad a la presencia de virus de transmisión sexual, corroborándose así que las normas tanto jurídicas, morales, entre otras, producen realidad, modelan conductas (disciplinan) y criminalizan mediante diferentes mecanismos de control. ${ }^{18}$

Como reacción a la liberación afectiva y sexual de los años de 1960, el conservadorismo volvió a representar la sexualidad como pecaminosa y peligrosa, ${ }^{19}$ actitud que incidió en el fortalecimiento de una cultura de autorrepresión a la afectividad que tradicionalmente por la visión colonial ha sido conectada con adjetivaciones sexuadas.

La afectividad y feminidad son por tanto potenciales y potentes motores de revolución social que, puestos en libertad, pueden dar origen a diversos tipos de familia no solo ventajosas para el sistema de producción privado, sino de muchos otros tipos de colaboración y organización humana horizontal rearmonizada.

\section{LA PROTECCIÓN JURÍDICA ESTATAL DE LA DIVERSIDAD DE FAMILIAS: CONSTITUCIÓN ECUADOR}

En Ecuador, el primer inciso del artículo 67 de su Constitución reconoce que la familia posee diversos tipos y que todos estos tipos de familia deben ser protegidos por el Estado, ya que todos los tipos de familia, tanto las surgidas por necesidad, las iniciadas desde el afecto, las que se conformaron desde la atracción sexual, las familias ampliadas u otras, son consideradas como núcleo fundamental de la sociedad; por ello, el Estado ecuatoriano debe garantizar todas aquellas condiciones que favorezcan no una parte sino integralmente la consecución de sus fines.

Entre otras cosas esto significa que las familias tanto monogámicas heterosexuales como las monogámicas homosexuales, las poliamorosas y muchas otras deben tener aseguradas por parte del Estado todas las condiciones para la consecución integral de sus fines; por lo tanto, la garantía de condiciones no aplica únicamente a la familia tradicional colonial o dominante que se encuentra en condiciones ventajosas frente al resto de tipos de familia.

18. Álvarez Mora y Monteros Obelar, Diversidad familiar: una perspectiva antropológica, 18.

19. Patricia de los Ríos, Los movimientos sociales de los años sesenta en Estados Unidos: un legado contradictorio (México: Universidad Autónoma Metropolitana, 1998), 26-8. 
Es claro entonces que el mandato constitucional expresa implícitamente que no puede continuar teniéndose una interpretación únicamente sexuada de la familia ya que las condiciones y relaciones entre las personas en tan diversa como diversas son las familias que de esas interrelaciones emergen pudiendo o no manifestarse en ellas elementos sexuales.

Así mismo, la Constitución ecuatoriana no deja duda de que no puede continuar pretendiéndose al modelo monogámico, monoamoroso y heterosexual como el modelo único, superior, universal o natural para mantener su posición de dominio no solo social sino jurídico que perpetúa a través de las normas estatales la homogenización social respecto al modelo de familia, y que deja en segundo plano o plenamente desprotegidos ante los actos de represión a las familias que existen y se desarrollan por fuera del estereotipo.

Ahora bien, en virtud del reconocimiento de existencia y la obligación de protección estatal para el desarrollo de la diversidad de familias, debe tenerse en cuenta que al hablar el mandato constitucional de garantizar las "condiciones que favorezcan integralmente la consecución de sus fines", está señalando la obligación y el compromiso del Estado de favorecer los fines incluso de las familias poliamorosas y comunitarias así como de aquellas que ven a la naturaleza como una madre o un miembro de la familia y que, por tanto, mantienen una relación especial de afectos y cuidados materiales mutuos con dicho miembro de la familia.

Es decir, dependiendo del tipo de familia, también los fines, las necesidades y aspiraciones de cada tipo de familia son diversos, tanto para el conjunto familiar como para cada uno de los miembros que la conforman, y cuyo bienestar hace parte del bienestar familiar, por lo que habrá familias con finalidades muy diferentes a las familias tradicionales coloniales.

Constitucionalmente, entonces, la familia tradicional colonial heterosexual monogámica no puede continuar utilizando al derecho como una herramienta de conservación del estatus para continuar arremetiendo contra las distintas formas en que la afectividad y el amor se manifiestan. Por el contrario, jurídicamente se encuentra abierto un camino para el desarrollo paulatino de reequilibrios entre la feminidad y la masculinidad capaz de comprenderse más allá del reducido esquema de dos individuos y sus hijos, ya que ha incluido la posibilidad de que las sociedades se comprendan como una gran y amplia familia que solidariamente redistribuye, frente al actual individualismo y falta de cooperación propio del esquema de pequeños grupos familiares enfrentados y destinados a competir.

En este aspecto, la Constitución ecuatoriana no es ajena a entender la manera cómo se distribuye el poder en equilibrio con el afecto con claro efecto en el ámbito socioeconómico; así se puede corroborar en el artículo 319, en el cual se reconocen diversas formas de organización de la producción en la economía, entre estas las comunitarias, 
cooperativas, empresariales públicas o privadas, asociativas, autónomas, mixtas y, además de ellas, las organizaciones familiares y domésticas.

Por lo tanto, una familia en donde los integrantes han logrado armonizar sus relaciones hacia la horizontalidad adquirirá la posibilidad de reproducir una organización de producción también de tipo horizontal, generándose un modelo de gestión de la economía familiar que va puliendo su oposición ante los patrones de dominación no solo en las relaciones intrafamiliares, sino también sociales, en especial si la familia se decanta por ser de tipo ampliada en lugar del modelo reduccionista y aislante de una familia monogámica.

Entonces, la protección jurídica que el Estado ecuatoriano está en la obligación de cumplir y hacer cumplir es la de garantizar todas las circunstancias y condiciones que fueren necesarias para que cualquier tipo de familia pueda lograr su desarrollo integral, que implica necesaria y forzosamente entender los objetivos del grupo social y de todos los sujetos que lo integran, así como asegurar el bienestar de todos los miembros de cada tipo de familia en un ambiente de respeto mutuo, libre de abusos o manipulaciones dominantes tanto externas como internas.

\section{CONCLUSIONES}

1. El modelo monogámico heterosexual, al haberse impuesto como único modelo jurídico y socialmente defendible, conllevó al sometimiento y a la represión de la diversidad de las expresiones afectivas, lo cual implicó un ataque incluyendo a la esfera jurídico-normativa contra la diversidad de familias que no encajaron dentro del esquema monogámico, monoamoroso y heterosexual.

2. La motivación para integrar familias es diversa y de ello deriva precisamente la diversidad de familias que se pueden originar. Estas motivaciones pueden ser sexuales, afectivas, de agremiación por necesidad, entre muchas otras, y en esta amplia diversidad incluso se puede anotar a aquellas familias amplias poliamorosas que gestionan en comunidad y cooperativamente las necesidades de sus miembros como, por ejemplo, sus descendientes y han incrementado su autosuficiencia y libre determinación. Así mismo, en esta amplia diversidad se incluyen aquellas familias que consideran la naturaleza o el ecosistema como un miembro de la familia que puede tener asignado diferente rango y valoración.

3. La relación entre los comportamientos asignados o identificados como masculinos y los identificados como femeninos se encuentra desequilibrada, priorizándose la masculinización y relegando o excluyendo la feminidad no solo en el ámbito familiar sino también en las interrelaciones sociales y en las estructuras institucionales, incluyendo los espacios científicos y profesionales. Esto ha 
conllevado a una falta de sensibilidad y a un afán de dominación en la manera en cómo se ejerce la autoridad y se toman decisiones.

4. Los parámetros reduccionistas y aislantes de la monogamia han favorecido el mantenimiento e impulso de un sistema de producción privado, ya que las familias se ven como grupos humanos extremadamente reducidos que compiten entre sí por la subsistencia y la propiedad, por lo que las sociedades han perdido oportunidades de organización cooperativa, libre determinación y felicidad al no poder verse solidariamente como hermanos o familias amplias.

5. Es obligación del Estado ecuatoriano, de acuerdo con su Constitución, garantizar que todo tipo de familias y no solo las monogámicas o las heterosexuales tengan condiciones suficientes para alcanzar sus objetivos de manera integral, de tal forma que el modelo convertido en común y dominante no continúe imponiéndose como el único susceptible de ser impulsado y protegido, de manera que se fomente entre otras cosas un proceso de rearmonización endo y exofamiliar. Y, al hablar de la garantía y obligación estatal para el alcance integral de los fines de cada tipo de familia, quienes estén revestidos de potestades estatales no pueden trasladar e imponer los mismos fines de las familias monogámicas heterosexuales a otras familias diversas y alternas a dicho modelo de afectos y relaciones, puesto que sus fines, necesidades e intereses son también distintos y como tales se encuentran incluidos en los parámetros y obligaciones de garantía para su desarrollo y alcance.

\section{BIBLIOGRAFÍA}

Álvarez Mora, Bruna, y Silvina Monteros Obelar. Diversidad familiar: una perspectiva antropológica. Barcelona: Editorial UOC, 2019.

Blanco L., O. Cedre, y M. E. Guerra. Factores determinantes del embarazo adolescente. Venezuela: Universidad Central de Venezuela, 2015.

Constitución de la República del Ecuador. 2008.

De los Ríos, Patricia. Los movimientos sociales de los años sesenta en Estados Unidos: un legado contradictorio. México: Universidad Autónoma Metropolitana, 1998.

Eroles Carlos, Angélico Héctor, y Alberto L. Bialakowsky. Familia(s), estallido, puente y diversidad: una mirada transdisciplinaria de derechos humanos. Buenos Aires: Espacio Editorial, 2006.

Fondo de las Naciones Unidas para la Infancia. Diez razones para poner fin legalmente al matrimonio infantil en República Dominicana. Santo Domingo: UNICEF, 2019.

Fong, Héctor, Dalila De la Cruz y Rocío López. Vidas robadas: repercusiones en la salud integral en las niñas menores de 14 años embarazadas. Guatemala: Observatorio de Salud Reproductiva, 2015. 
García Daniel. Protesta y política: los movimientos antiguerra en Estados Unidos. Bogotá: Universidad de los Andes, 1988.

Gastiz, Victoria. Los hombres, la igualdad y las nuevas masculinidades. Araba: Instituto Vasco de la Mujer Emakunde, 2008.

Martínez William. "Matrimonios a la fuerza". El Tiempo (Colombia). 5 de marzo de 2000.

Mazzei Claudia. Producción y reproducción: la mujer y la división socio-sexual del trabajo. Chile: Universidad Central de Chile RUMBOS, 2013.

Organización Mundial de la Salud. El embarazo en adolescentes. Washington: OMS, 2015.

-. The health andwell-being of men in theWHO European Region: better health througha gender approach. United Kingdom: OMS, 2018.

Pascual, Belén. Masculinidades: ¿por qué los hombres también necesitan feminismo? Castellón: Universidad de Jaume, 2015.

Polo, Sara. "Poliamor". El Mundo. 12 de febrero de 2016.

Sandoval, Ana Elisa. La licuefacción de la sexualidad: una aproximación a la pansexualidad en la modernidad líquida. Quito: Pontificia Universidad Católica del Ecuador, 2016.

Tapia Paredes, José Ernesto. "Perspectivas para la protección normativa de los conocimientos tradicionales frente al régimen de propiedad intelectual en el comercio internacional". Tesis de maestría, Universidad Andina Simón Bolívar, Sede Ecuador, 2014. 